DESY $94-178$

October 1994

Deep Inelastic Structure Functions from HERA

G. Wolf

Deutsches Elektronen-Synchrotron DESY, Hamburg $\sin 3444$

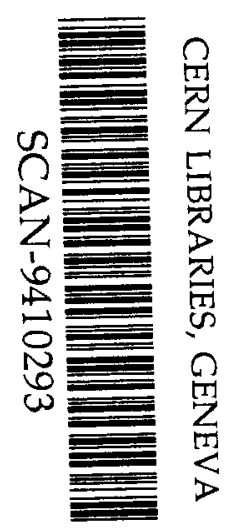




\title{
Deep Inelastic Structure Functions from HERA*
}

\author{
Günter Wolf \\ 11 \\ Deutsches Elektronen-Synchrotron, DESY, \\ Notkestrasse 85, 22603 Hamburg, Germany
}

Results on deep inealstic scattering obtained by $\mathrm{H} 1$ and ZEUS from the 1993 data taken at HERA are presented. The measurements of the charged current process provide first evidence for the damping of the total neutrino cross section at high energies by the W propagator. A comparison with neutral current scattering shows that at $Q^{2}$ values above the $W$ mass squared the weak cross section becomes comparable to the electromagnetic one. The structure function $F_{2}$ of the proton was determined from neutral current scattering data. The results confirm the steep rise of $\mathrm{F}_{2}$ towards small $\mathrm{x}$ below $10^{-2}$ seen previously by the two experiments albeit with much less statistics. The rise persists from the lowest $Q^{2}$ values up to $500 \mathrm{GeV}^{2}$. The $F_{2}$ data are in accord with logarithmic scaling violations in a new regime of low $x$ and high $Q^{2}$. An analysis of $F_{2}$ data by ZEUS in terms of the total virtual photon proton cross section shows $\sigma_{\text {to }}\left(\gamma^{*} p\right)$ to increase with the total $\gamma^{*}$ p c.m. energy, the increase being compatible with a linear rise. This is in striking contrast with the behaviour of the total cross section for real photons or for pp scattering.

\section{INTRODUCTION}

The high centre of mass energy available at HERA presents a vast increase in phase space for lepton - nucleon scattering in comparison with fixed target experiments. The maximum $Q^{2}$ values that can be reached are two orders of magnitude larger while for fixed $\mathrm{Q}^{2}$ the minimum Bjorken-x values are two orders of magnitude smaller.

The first measurements performed by $\mathrm{H} 1$ [1] and ZEUS [2] on neutral current scattering showed the proton structure function, $F_{2}$, to exhibit a strong rise as $x$ decreases below $10^{-2}$. New results on $F_{2}$ were obtained from data taken in 1993. They correspond to a twentyfold increase in statistics and strengthen the conclusion that at small values of $x$ a new regime of physics is entered.
Charged current scattering has a much smaller cross section and therefore produces correspondingly less events. Both experiments had a first look at this type of process with the 1993 data.

\subsection{HERA}

In 1993 HERA operated with 84 electron bunches of $26.7 \mathrm{GeV}$ colliding with 84 proton bunches of $820 \mathrm{GeV}$, with a bunch spacing between crossing of $96 \mathrm{~ns}$. In addition, there were 10 unpaired electron and 6 unpaired proton bunches for the study of background. Typical beam currents were $10-15 \mathrm{~mA}$ yielding a luminosity of $0.7 \cdot 10^{30} \mathrm{~cm}^{-2} \mathrm{~s}^{-1}$. The total integrated luminosity per experiment reached $600 \mathrm{nb}^{-1}$ compared to $33 \mathrm{nb}^{-1}$ in 1992. During the present running period (1994), a further increase of the luminosity by a factor $4-5$ will be achieved.

* Invited talk given at the XVI Intern. Conf. on Neutrino Physics and Astrophysics, Eilat, Israel, May 29 - June 3, 1994. 


\subsection{The $\mathrm{HI}$ and ZEUS detectors}

In figs. 1,2 displays of events from charged and neutral current scattering from the $\mathrm{H} 1$ and ZEUS experiments are shown. The principal detector components used for the analysis of the data presented below were the central tracking systems, the calorimeters and the luminosity detectors.

In the $\mathrm{H} 1$ experiment, the calorimeter is made of lead and iron absorber and read out with liquid argon in the forward and barrel region and scintillator in the rear. The liquid argon calorimeter (LAC) covers the angular region $4^{\circ}<\theta<153^{\circ}$ ( the forward region, $\theta=$ 0 , is given by the direction of the proton beam). It is longitudinally subdivided into several electromagnetic and hadronic sections and is segmented into a total of 45000 cells. The energy resolution $\sigma / \mathrm{E}$ for electrons is $12 \% / \sqrt{\mathrm{E}} \oplus 1 \%$ ( $\mathrm{E}$ in $\mathrm{GeV}, \oplus$ means quadratic addition) and $50 \% / \sqrt{ } \mathrm{E} \oplus 2 \%$ for hadrons (after weighting), as measured with test beams. The calorimeter in the rear $\left(151^{\circ}<\theta<177^{\circ}\right)$ serves as an electromagnetic calorimeter and is followed by time-of-flight counters.

The ZEUS detector uses a uranium-scintillator calorimeter covering polar angles of $1.6^{\circ}<\theta<177.4^{\circ}$. The calorimeter is longitu- dinally subdivided into an electromagnetic and two (one) hadronic sections in the forward and barrel (rear) parts and is segmented into 5918 cells each read out by two photomultipliers. The energy resolution found in test beams is $18 \% / \sqrt{\mathrm{E}}$ for electrons and $35 \% / \sqrt{ } \mathrm{E}$ for hadrons. The calorimeter provides also a time measurement. The time resolution of a cell is $\sigma=1.4 / \mathrm{E}^{0.65} \oplus 0.4 \mathrm{~ns}$, where $\mathrm{E}$ (in $\mathrm{GeV}$ ) is the energy deposited in the cell.

The luminosity is measured in both experiments by observing the bremsstrahlung process $e p \rightarrow e^{i} p \gamma$ at very small angles to the electron beam direction. The final state electron and photon are detected in electromagnetic calorimeters located about $33 \mathrm{~m}$ and $105 \mathrm{~m}$ upstream of the collision point in the proton direction. The rate of luminosity events was typically several $\mathrm{kHz}$.

\section{KINEMATICS}

Deep inelastic scattering (DIS), e p $\rightarrow \mathrm{l}^{\prime}+$ anything, as depicted in fig. 3 will be described in terms of the variables shown in Table 1 .

For neutral current reactions (NC), $\mathrm{e}^{-} \mathbf{p} \rightarrow \mathrm{e}^{-}$ $+\mathrm{X}$, due to the almost complete solid angle coverage of the HERA detectors, the event

Table 1

Definition of kinematic variables for deep inelastic electron proton scattering

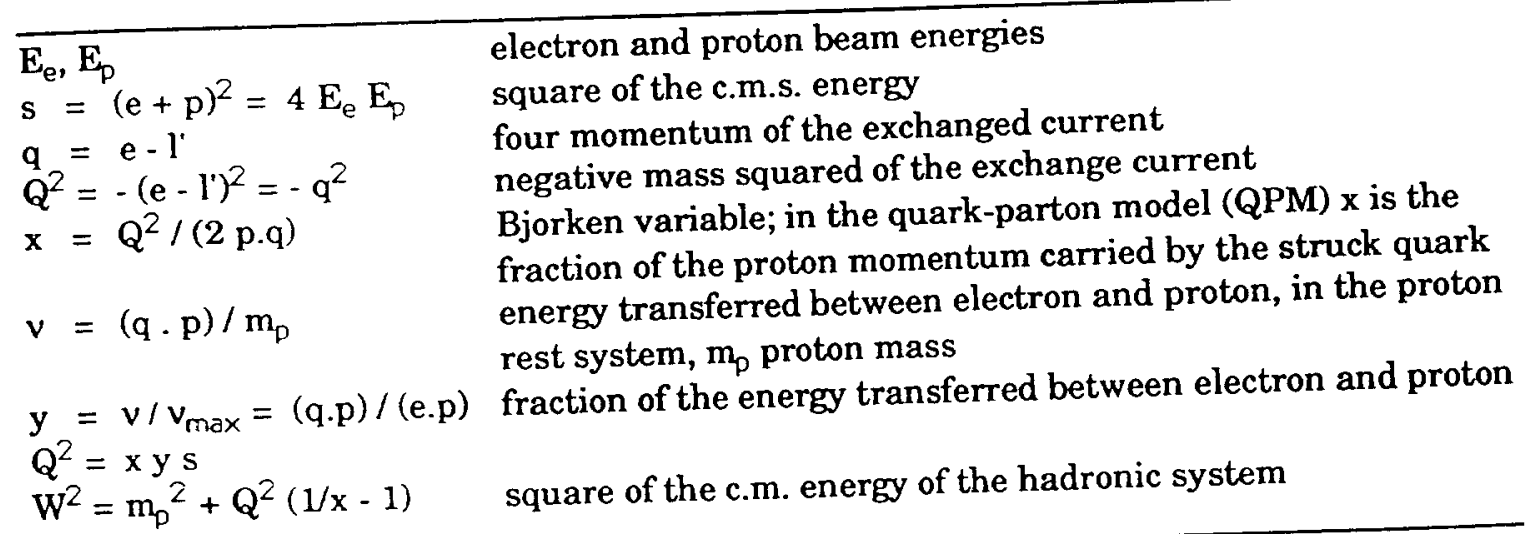



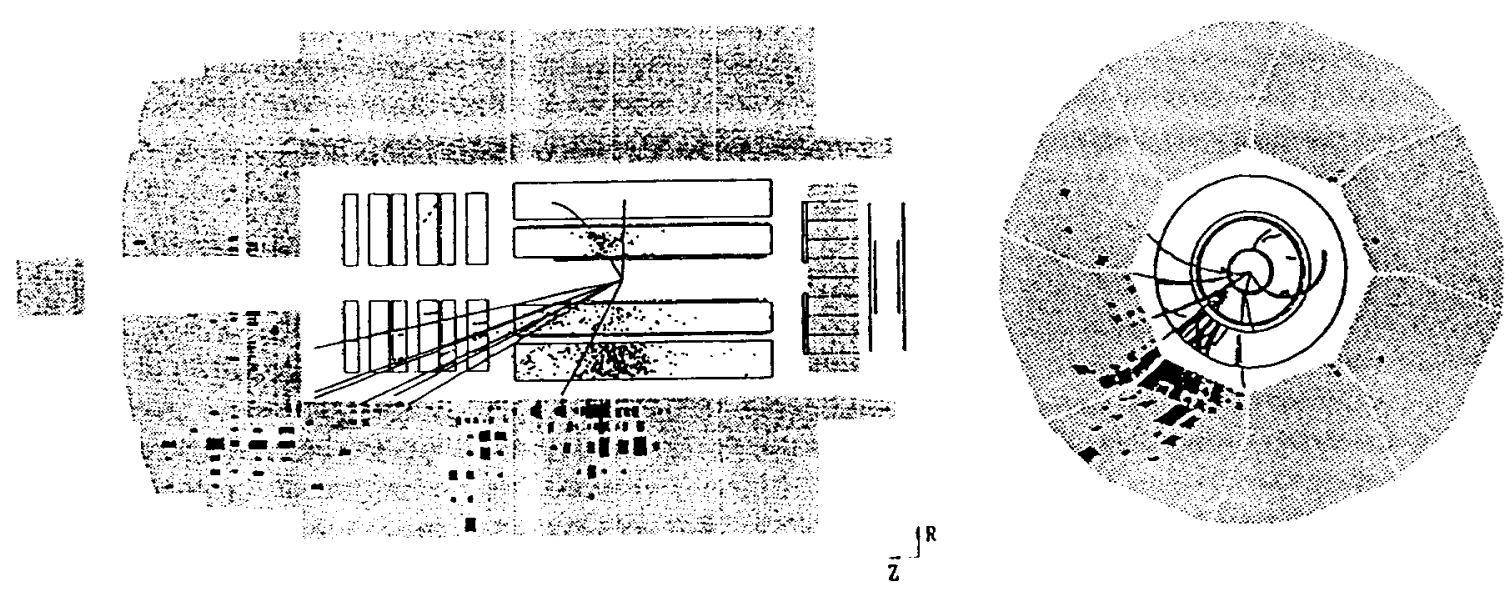

$\vec{x}^{Y}$

Figure 1. A charged current event with $\mathrm{Q}^{2}=23400 \mathrm{GeV}^{2}, \mathbf{p}_{\mathrm{T}}^{\text {miss }}=59 \mathrm{GeV}$ as seen in the $\mathrm{H} \mathbf{1}$ detector.

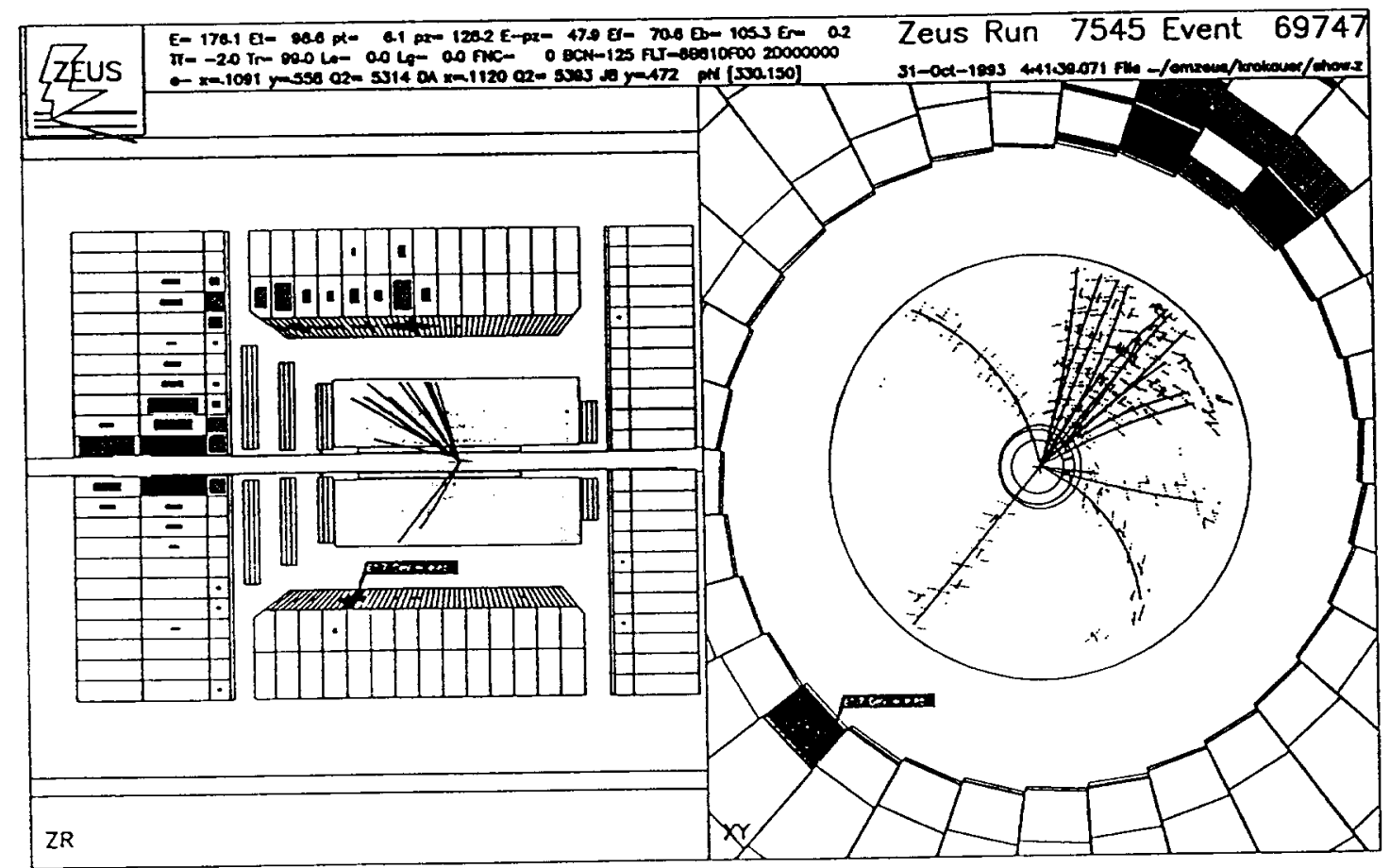

Figure 2. A neutral current event with $\mathrm{Q}^{2} \approx 5300 \mathrm{GeV}^{2}, \mathrm{x} \approx 0.11$ as seen in the ZEUS detector. 


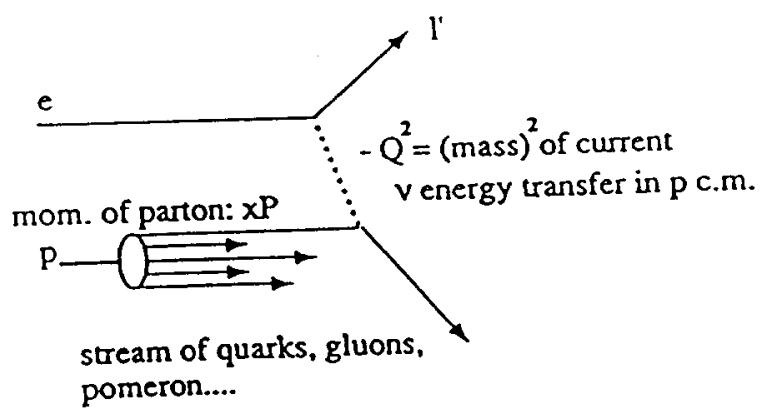

Fig.3 Diagram for ep scattering

kinematics can be determined from the energy and angle of either the scattered electron or of the produced hadronic system, or from a combination of these. For instance, y calculated from the electron variables is given by

$\mathrm{y}_{\mathrm{e}}=1-\left(\mathrm{E}_{\mathrm{e}} / 2 \mathrm{E}_{\mathrm{e}}\right)\left(1-\cos \theta_{\mathrm{e}}{ }^{\prime}\right)$

where $E_{e}{ }^{\prime}, \theta_{e}$ are the energy and polar angle of the scattered electron. The variables $y$ and $\mathrm{Q}^{2}$ (and also $\mathrm{x}$ ) can be determined from the hadronic system as measured by the calorimeter, using the Jacquet-Blondel technique [3]:

$y_{J B}=\Sigma_{h}\left(E_{h}-p_{A h}\right) /\left(2 E_{e}\right)$
$Q_{B}{ }^{2}=\left[\left(\Sigma_{h} p_{X h}\right)^{2}+\left(\Sigma_{h} p_{x h}\right)^{2}\right] /\left[1-y_{J B}\right]$

where $p_{x h}=E_{h} \sin \theta_{h} \cos \phi_{h}, p_{y h}=E_{h} \sin$ $Q_{h} \sin \phi_{h}, p_{\hbar h}=E_{h} \cos \theta$ and $E_{h}$ is the energy measured in calorimeter cell $h$ with polar and azimuthal angles $\theta_{h}, \phi_{h}$ as determined from the coordinates of the cell and the event vertex. The summation is performed over all calorimeter cells. The method rests on the assumption that the total transverse momentum carried by those hadrons escaping through the forward beam hole can be neglected as well as the energy and momentum of particles escaping through the rear beam hole.

In the double-angle (DA) method [4] $x$ and $Q^{2}$ are determined from the angles of the scattered electron $\left(\theta_{e}{ }^{\prime}\right)$ and the produced hadron system $\left(\theta_{n}\right)$. The method relies on ratios of energies and therefore is less sensitive to scale uncertainties of the energy measurement.

The increase of phase space provided by HERA for lepton nucleon scattering in comparison with present fixed target experiments is illustrated in Table 2.

Table 2

Kinematic region accessible at $\operatorname{HERA}\left(\mathrm{E}_{\mathrm{e}}=26.7 \mathrm{GeV}, \mathrm{E}_{\mathrm{\rho}}=820 \mathrm{GeV}\right)$ and in fixed target experiments

HERA fixed target experiments

$\begin{array}{lll}\mathrm{s}\left(\mathrm{GeV}^{2}\right) & 0.9 \cdot 10^{5} & 10^{3} \\ \text { maximum practical } \mathrm{Q}^{2}\left(\mathrm{GeV}^{2}\right) & 40000 & 400 \\ v_{\max }(\mathrm{GeV}) & 47000 & 500 \\ \text { minimum } \times \text { at } \mathrm{Q}^{2}=10 \mathrm{GeV}^{2} & 1 \cdot 10^{-4} & 1 \cdot 10^{-2}\end{array}$




\section{CHARGED CURRENT SCATTERING}

Charged current (CC) processes of the type

$$
\text { e p } \rightarrow \text { v X }
$$

can be detected via missing transverse momentum, $p_{\top}{ }^{\text {miss }}$, carried away by the neutrino.
The main parameters of the $H 1$ [5] and ZEUS [6] analyses are summarized in Table 1. The ZEUS data are still preliminary. Both experiments are able to isolate with high efficiency a background free sample of $\mathrm{CC}$ events by selecting events on $\mathrm{p}_{\mathrm{T}}{ }^{\text {miss }}$, by requiring a tracking vertex, by recognizing events produced by muons accompanying the proton beam or due to cosmic rays and/or superimposed on an event from ep collisions.

Table 1

Selection of charged current events: the numbers of remaining candidates are underlined

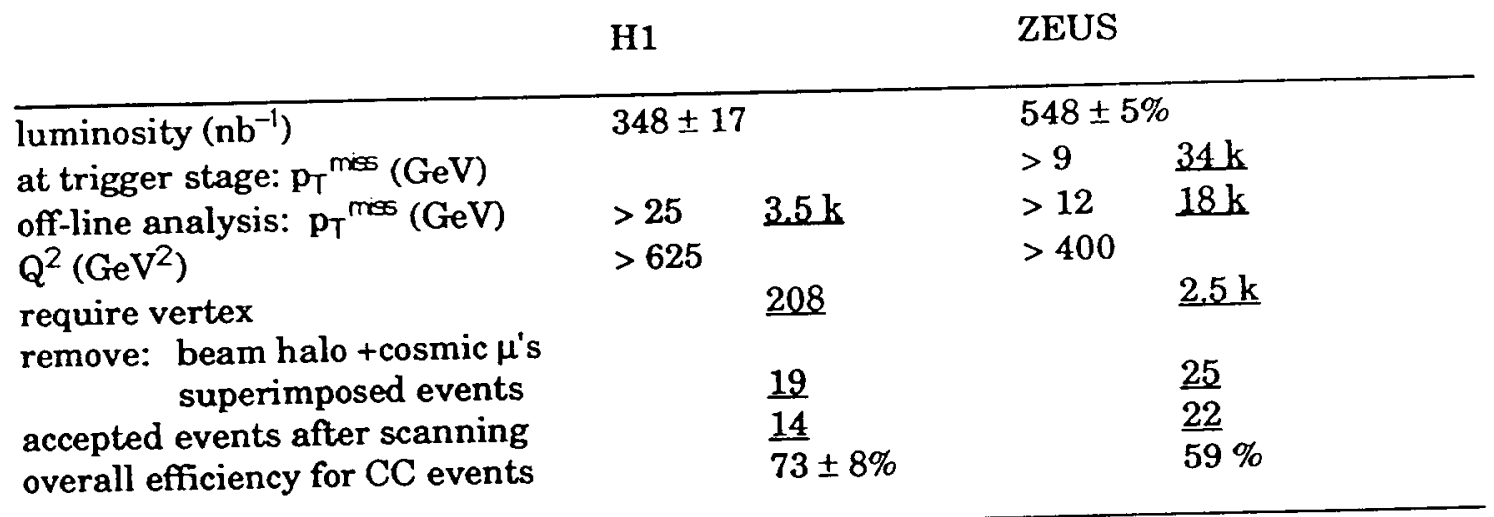

The effect of the $\mathbf{p}_{\top}{ }^{\text {miss }}$ selection on the background in the $\mathrm{H} 1$ analysis is illustrated in Fig. 4 which shows the distribution of the scalar (S) versus vector $(\mathrm{V})$ transverse momentum sums defined as:

$$
\begin{aligned}
& \mathrm{S}=\Sigma_{i}\left|\overline{\mathrm{p}}_{T i}\right| \\
& \mathrm{V}=\left|\Sigma_{i} \overline{\mathrm{p}}_{T i}\right| \equiv \mathrm{p}_{T}{ }^{\text {miss }}
\end{aligned}
$$

where $\bar{p}_{T i}$ are the momentum vectors in the plane transverse to the beams determined from the calorimeter cell information. By definition $\mathrm{S} \geq \mathrm{V}$. $\mathrm{CC}$ events are characterized by large values of $S$ and $T$. The background (mainly beam related) at large $S$ but small V is suppressed by requiring $V \equiv p_{T}^{\text {miss }}>25$ GeV (see Fig. 4).

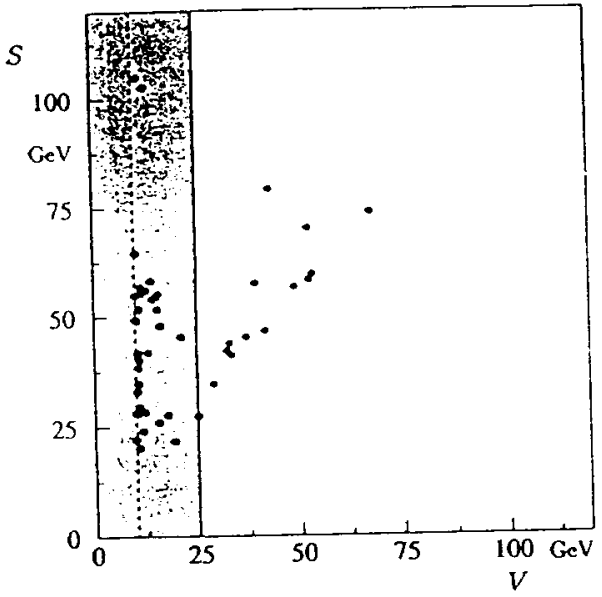

Figure 4. Selected events in the V - S plane for $\mathrm{V}>10 \mathrm{GeV}$. The signal region is defined by $\mathrm{V}>25 \mathrm{GeV}$. (From $\mathrm{H} 1$ ). 
Figure 5 shows the total neutrino cross section averaged over protons and neutrons as a function of the neutrino energy $E_{v}$ measured in the nucleon rest system. The data up to about $\mathrm{E}_{\mathcal{v}}=25 \mathrm{GeV}$ were measured in fixed target experiments. They show a linear rise of the cross section with $\mathrm{E}_{\mathcal{V}}$ consistent with an infinitely heavy $W\left(m_{W}=\infty\right)$. The result from $\mathrm{H1}$ is

$$
\begin{aligned}
\sigma\left(\mathrm{e}^{-\mathrm{p}}\right. & \left.>\mathrm{v}+\text { hadrons, } \mathrm{p}_{\mathrm{T}}>25 \mathrm{GeV}\right) \\
= & 55 \pm 15 \text { (stat) } \pm 6 \text { (syst) } \mathrm{pb}
\end{aligned}
$$

The data point at $E_{v}=46 \mathrm{TeV}$ of fig. 5 shows the $H 1$ result converted into a $\vee N$ cross section. It iies well below the straight line obtained from the extrapolation of the low energy data and demonstrates the effect of the W propagator. Figure 6 shows directly the sensitivity of the $\mathrm{H} 1$ measurement to the mass of the $W$ propagator: The case of an infinitely heavy W (dashed line) is excluded by the data.

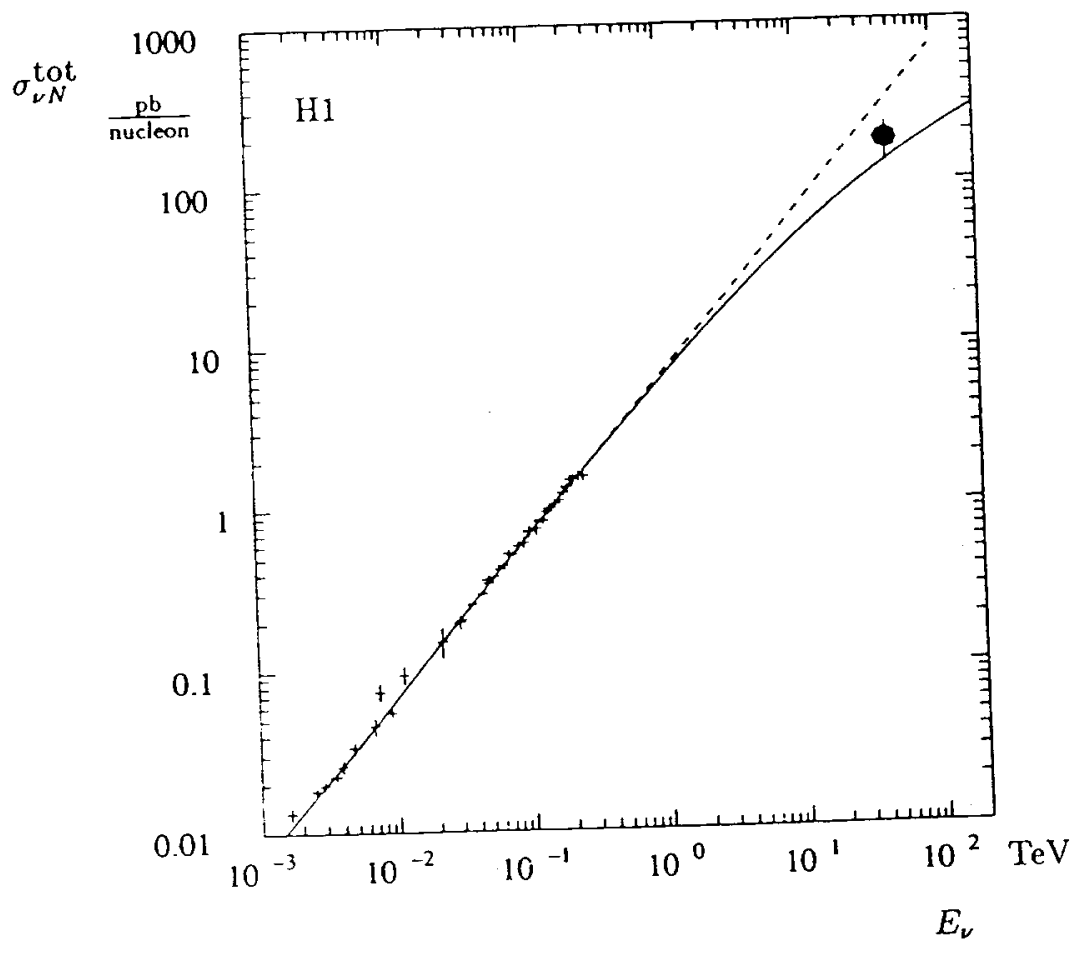

Figure 5. The energy dependence of the $v \mathrm{~N}$ cross section as a function of $\mathrm{E}_{v}$ measured in the nucleon rest system. The crosses represent the low energy neutrino data. The point at $46 \mathrm{TeV}$ shows the $\mathrm{H} 1$ result. The dashed line shows the linear extrapolation of the low energy data; the solid line shows the predicted cross section taking the W mass into account. (From H1). 


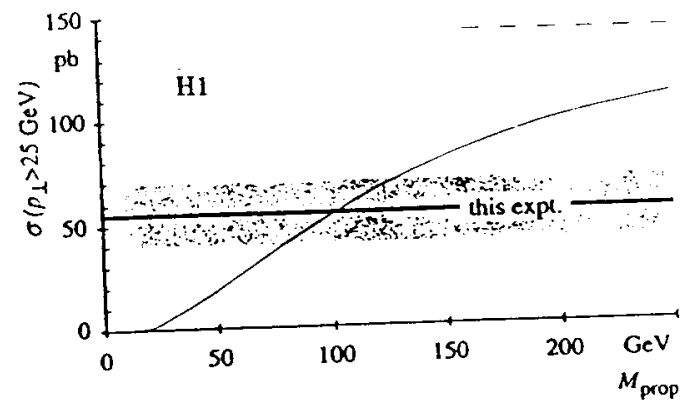

Figure 6. The $\mathrm{H} 1$ result for the $\mathrm{CC}$ cross section (= horizontal line with \pm 1 s.d. band) compared with the cross section predicted as a function of the propagator mass. The dashed line indicates the expectation for $\mathrm{M}_{\text {prop }}=\infty$. (From H1).

The ZEUS group analyzed simultaneously $\mathrm{CC}$ and NC scattering. A critical part of the analysis was the determination of $x$ and $Q^{2}$ for the CC events. While for NC events these variables can be measured from either the electron or the hadron side, thereby allowing for a cross check, for CC events only the hadron side is accessible for measurement. The values of $x$ and $Q^{2}$ were determined with the Jacquet-Blondel method. The accuracy of the method and the necessary corrections were determined independently with the help of the NC events and by Monte Carlo simulations. Consistent results were obtained. The $x-Q^{2}$ distribution of the $\mathrm{CC}$ events is shown in Fig. 7. The highest $\mathrm{Q}^{2}$ value is above $20000 \mathrm{GeV}^{2}$.

The differential CC cross section

$d \sigma / d^{2}\left(e-p-v X, x_{a}-x_{b}\right)$

integrated over the $x$-bins $x_{a}-x_{b}$ indicated in Fig. 7 is shown in Fig. 8. Also given in Fig. 8 is the NC cross section, $\mathrm{d} \sigma / \mathrm{dQ}^{2}\left(\mathbf{e}^{-} \mathrm{p}-\mathrm{eX}, \mathrm{x}_{\mathrm{a}}-\mathrm{x}_{\mathrm{b}}\right)$

determined for the same $x$-bins. While at $Q^{2}$ $=400 \mathrm{GeV}^{2}$ the NC cross section is two orders of magnitude larger than the CC cross section, one sees for the first time that at $Q^{2}$ values of the order of the $W$ mass squared the weak cross section becomes comparable to the electromagnetic one.

The solid lines in Fig. 8 show the QCD predictions for the $\mathrm{CC}$ and NC cross sections. The prediction of an infinitely heavy $W$ (dashed line) is ruled out by the CC data.

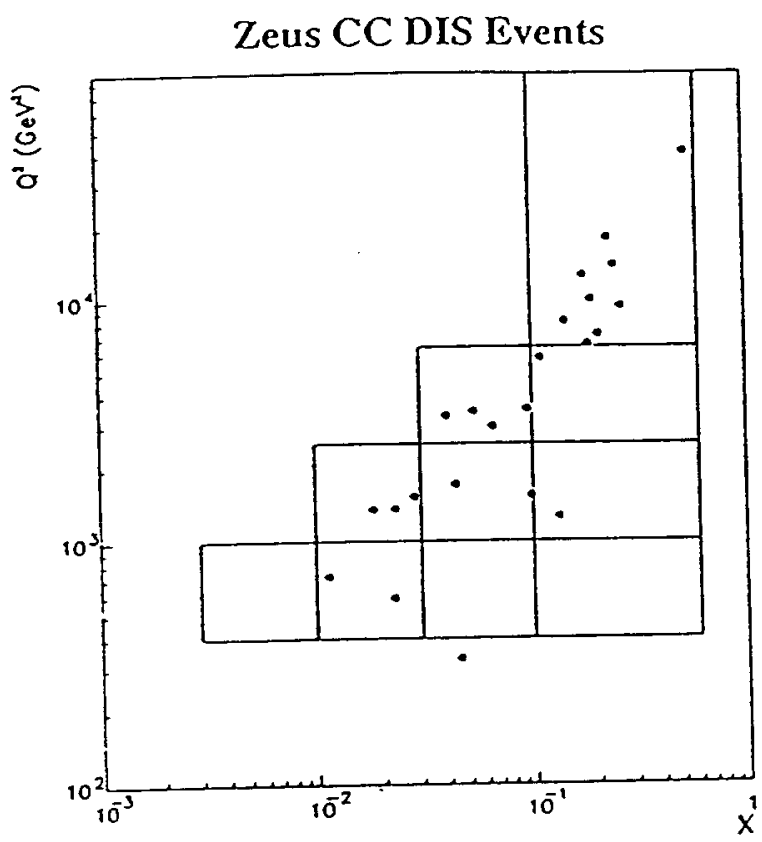

Figure 7. Distribution of CC events in the $x-Q^{2}$ plane. (From ZEUS). 


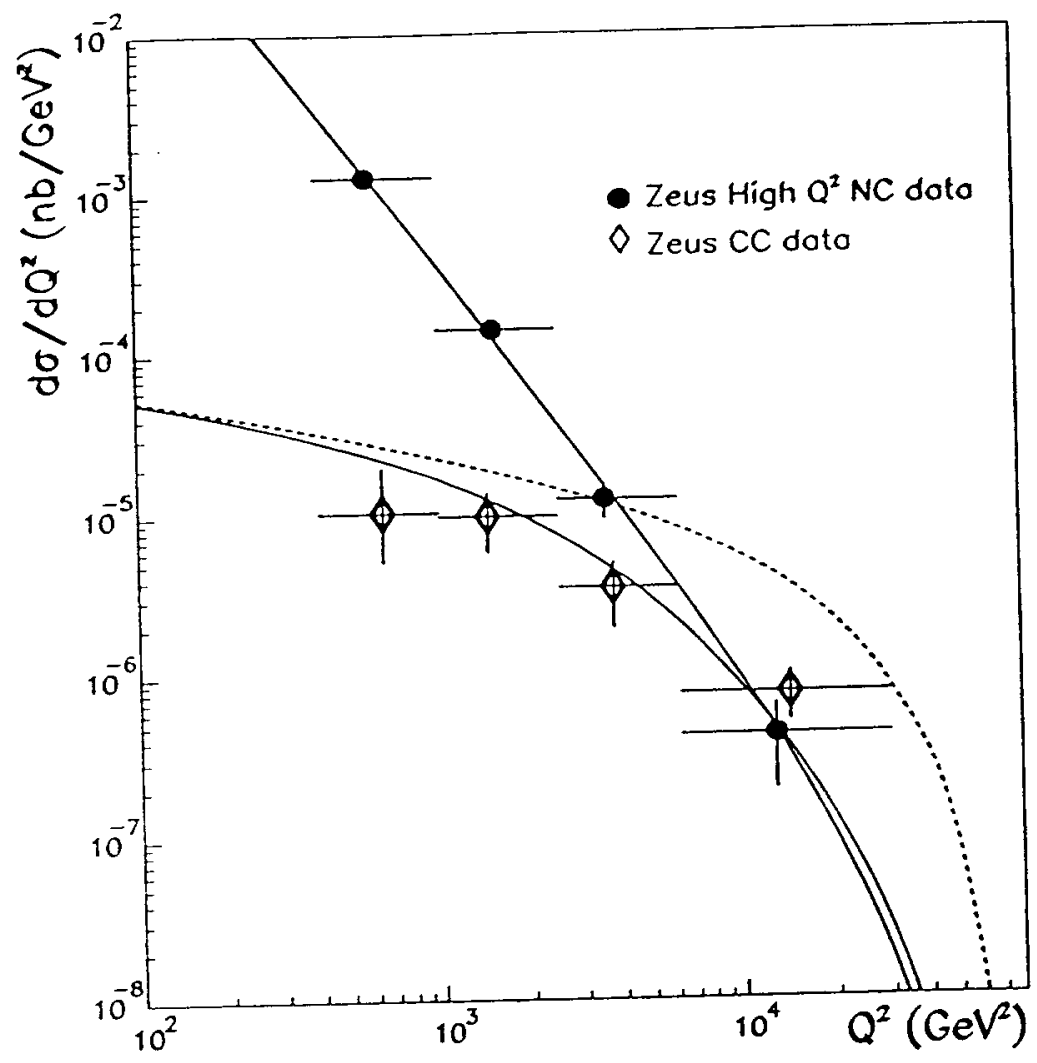

Figure 8. The cross sections $\mathrm{d} \sigma / \mathrm{dQ}^{2}$ for $\mathrm{NC}$ (full dots) and $\mathrm{CC}$ scattering (open dots) integrated over the x-bins indicated in Fig. 7. The solid curves show the QCD predictions calculated with the structure function set MRSD-' [7]. The dashed curve shows the prediction for the CC cross section for an infinite W mass. (From ZEUS).

\section{NEUTRAL CURRENT SCATTERING}

The large event statistics obtained from neutral current scattering

$$
\mathrm{e}^{-p} \rightarrow \mathrm{e}^{-} \mathrm{X}
$$

allowed both experiments to embark on a precision measurement of $F_{2}$ at low $x$. As mentioned before, the kinematical variables $x$ and $Q^{2}$ can be determined in different ways, using various combinations of the electron and hadron measurements with different experimental and theoretical uncertainties (e.g. electromagnetic radiative corrections). Performing the analyses with these different combinations provided important cross checks of the results.

The main and only important source of background was photoproduction where in general the scattered electron disappeared through the rear beam hole and another particle was considered in its stead. The variable $\delta$, defined as 


$$
\delta=\Sigma_{i}\left(\mathrm{~F}_{\mathrm{i}}-\mathrm{p}_{\mathrm{j}}\right),
$$

where the summation includes also the energy deposit by the electron, was used to suppress photoproduction events and to estimate from its distribution the remaining photoproduction background. For fully contained events energy-momentum conservation requires $\delta=2 \mathrm{E}_{\mathrm{e}}(=53.4 \mathrm{GeV}$ for the present data). The loss of particles through the forward beam hole in general has only a small effect on $\delta$ since $E_{\bar{y}} \approx p_{j z}$. For photoproduction, on the other hand, the loss of the scattered electron leads to significantly smaller values of $\delta$.

\subsection{Event selection}

The main parameters of the $H 1$ [8] and ZEUS [9] analyses are given in Table 3 . The H1 data are still preliminary.

\section{Table 3}

Selection of neutral current events. The index $e(h)$ indicates electron (hadron system) variables. The $\sum$ method combines electron and hadron energy flows.

H1

\begin{tabular}{lll}
\hline luminosity $\left(\mathrm{nb}^{-1}\right)$ & 270 & $543 \pm 3.3 \%$ \\
number of events & $\sim 20000$ & 46000 \\
$\mathrm{x}, \mathrm{Q}^{2}$ determined from & $\mathrm{x}_{\mathrm{e}}, \mathrm{Q}_{\mathrm{e}}^{2} ; \mathrm{Q}_{\mathrm{e}}^{2}, \mathrm{y}_{\mathrm{JB}} ;$ & $\mathrm{x}_{\mathrm{e}}, \mathrm{Q}_{\mathrm{e}}^{2} ; \theta_{\mathrm{e}}, \theta_{\mathrm{h}}$ \\
& $\theta_{\mathrm{e}}, \theta_{\mathrm{h}} ; \sum$ method & \\
some selection cuts & $\mathrm{E}_{\mathrm{e}}>8 \mathrm{GeV}, \mathrm{y}_{\mathrm{e}}<0.8$ & $\mathrm{E}_{\mathrm{e}}{ }^{\prime}>5 \mathrm{GeV}, \mathrm{y}_{\mathrm{e}}<0.95$ \\
& & $\mathrm{y}_{\mathcal{}}>0.04$
\end{tabular}

The event distributions in the $\mathrm{x}-\mathrm{Q}^{2}$ plane are shown in Figs. 9, 10 for $\mathrm{H} 1$ and ZEUS. Also indicated in Fig. 10 are the kinematic regions where data from fixed target experiments exist. Events with $Q^{2}$ values up to $20000 \mathrm{GeV}^{2}$ were observed. The suppression of events at low $Q^{2}$ is due to the requirement that scattered electrons detected in the rear calorimeter be sufficiently away from the beam line. In the case of ZEUS, the lack of events at low y values is a result of the cut $y_{\mathcal{B}}<0.04$ which insures the applicability of the DA - method.

\section{ZEUS}

\subsection{The structure function $\mathrm{F}_{2}$}

The NC cross section was determined for the $x-Q^{2}$ bins indicated in Figs. 9,10. The observed number of events was corrected bin-by-bin for the remaining background. Corrections for acceptance, detector smearing and radiative effects were determined by Monte Carlo simulations where the generated events were passed through the detector and selection procedures identical to those used for the real data. The NC cross section can be expressed in terms of three structure functions, $F_{2}, F_{L}$ and $x F_{3}$ : 


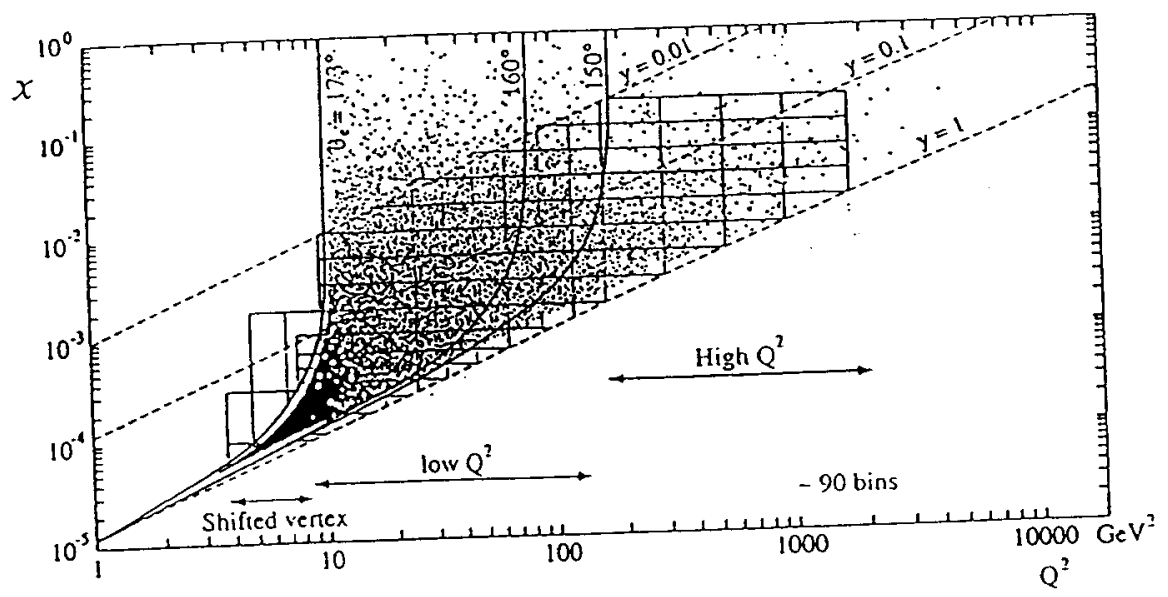

Figure 9. Distribution of the NC event sample from $H 1$ in the $x-Q^{2}$ plane.

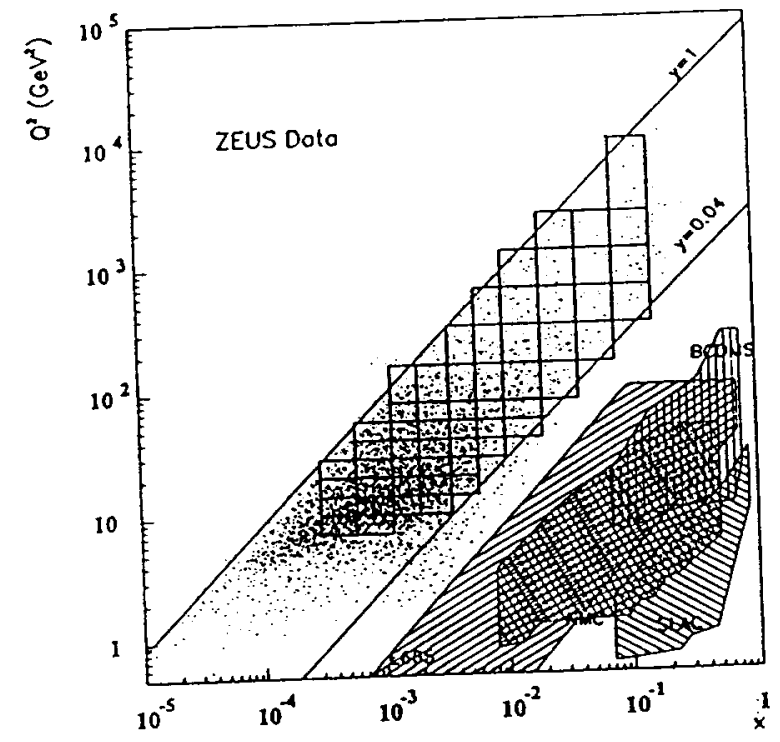

Figure 10. Distribution of the NC event sample from ZEUS in the $x-Q 2$ plane.

$$
\begin{aligned}
\frac{d^{2} \sigma}{d x d Q^{2}}= & \frac{4 \pi \alpha^{2}}{x Q^{4}}\left\{\left(1-y+y^{2} / 2\right) F_{2}\left(x, Q^{2}\right)\right. \\
& -y^{2} / 2 F_{L}\left(x, Q^{2}\right) \\
& \left.+\left(y-y^{2} / 2\right) x F_{3}\left(x, Q^{2}\right)\right\}\left[1+\delta_{4}\right]
\end{aligned}
$$

The $F_{3}$ term measures parity violating contributions which arise from $\mathrm{Z}^{0}$ exchange. They are significant only when $Q$ is comparable to or larger than the $Z^{0}$ mass. $F_{L}$ is the longitudinal structure function; or is the radiative correction. The familiar ep structure functions which receive only contributions from the exchange of a virtual photon are denoted by $F_{2}$ and $F_{L}$. Since the $Z^{0}, F_{L}$ and radiative corrections are small in all bins, eq. (1) can be rewritten as

$$
\begin{gathered}
\frac{d^{2} \sigma}{d x d Q^{2}}=\frac{4 \pi \alpha^{2}}{x Q^{4}}\left\{\left(1-y+y^{2} / 2\right) F_{2}\left(x, Q^{2}\right)\right. \\
\left..\left(1-\delta_{F L}+\delta_{Z}\right) \cdot\left(1+\delta_{r}\right)\right\}
\end{gathered}
$$


where the corrections $\delta_{q}$ are functions of $x$ and $Q^{2}$ but to a good approximation independent of $F_{2}$.

In the $\mathrm{H} 1$ and ZEUS analyses $\mathrm{F}_{2}$ was extracted from the data. Corrections were made for the contribution from $Z^{0}$ which was calculated using the MRSD-' parton densities and GLAP [10] QCD evolution. At the highest mean $\mathrm{Q}^{2}$ of $4200 \mathrm{GeV}^{2}$ the correction amounted to $20 \%$ relative to the $\mathrm{F}_{2}$ contribution; at the next lower $Q^{2}$ bin it was already less than $6 \%$. The correction for the contribution from $F_{L}$ was determined in the same way; it was found to be at the percent level relative to the $F_{2}$ contribution except for some low - $x$ bins where it amounted to 12 $15 \%$. ZEUS found the radiative corrections to be $3-5 \%$ for the double angle method and at most $10 \%$ if only electron variables were used.

The analyses performed by both experiments with different combinations of electron and hadron variables for the determination of $x$ and $Q^{2}$ produced $F_{2}$ values which were consistent within the systematic errors. ZEUS chose the results obtained with the DA method as the final values for $F_{2}$ while $H 1$ used the data obtained from the scattered electron and the $\Sigma$ method.

Figure 11 shows the data from ZEUS and $\mathrm{H} 1$ as a function of $x$ for fixed $Q^{2}$ values between 8.5 and $2000 \mathrm{GeV}^{2}$. The error bars give the statistical and systematic errors added in quadrature. There is good agreement between the two experiments. The data exhibit below $x=10^{-2}$ a strong rise of $F_{2}$ as $x$ decreases, a feature which was already observed in the 1992 HERA data $[1,2]$ but which now is seen with much higher statistics. This behaviour is in striking contrast to the almost constant behaviour seen at larger $x$ values. The steep rise persists from the lowest $Q^{2}$ values up to $Q^{2}$ of $500 \mathrm{GeV}^{2}$.

In Fig. 12 the $\mathrm{F}_{2}$ measurements from $\mathrm{H} 1$ and ZEUS are shown as a function of $Q^{2}$ for fixed values of $x$. Also included are data from
NMC [11] and preliminary data from E665 [12] obtained at smaller $Q^{2}$ values. The data are in accord with the logarithmic scale breaking expected from QCD, now measured in a new regime of high $Q^{2}$ and low $x$.

For theory the rise of $F_{2}$ was not unexpected. In pertubative $Q C D F_{2}$ was shown to grow faster than any power of $\ln (1 / x)$ as $x \rightarrow 0[13$, 14] and the qualitative feature of a rapid rise was anticipated by calculations in which only valence-like distributions of partons were evolved from very small $Q^{2}$ [15]. The rise is the manifestation of a rapidly growing density of partons in the proton as $x$ tends to zero. According to Lipatov [16] the gluon density in the proton should rise as $\mathbf{g}(\mathbf{x})$ $x^{-(1+\lambda)}$ for $x \rightarrow 0$, where $\lambda \approx \alpha_{5}[12 \ln 2] / \pi=$ 0.5 .

The measured $F_{2}$ data are compared in Figs. 11,12 with different extrapolations and a prediction, all based on the GLAP QCD evolution equations and on data measured at larger $x$ values. The curves MRSD- and MRSDo' [7] represent roughly the extremes of the extrapolations. At the scale where the evolution starts, $Q_{0}^{2}=4 \mathrm{GeV}^{2}, D^{2}$ has a singular parametrisation for the sea quark and gluon densities, $x f(x) \sim x^{-n}$ as $x \rightarrow 0$ where $\mathbf{n}=0.5$, and $\mathrm{Do}^{\circ}$ has a constant behaviour, $n=0$. At the lowest values of $Q^{2}$ these two parton densities (PDF) span the HERA data; for $Q^{2} \geq 35 \mathrm{GeV}^{2}$ the $\mathrm{D}$ - curves agree with the data. CTEQ2D' [17] assumes also a singular gluon distribution. It is typical of recent PDF evaluations (see e.g. MRSA [18]) that have included HERA data among the data sets used to determine their parameters and that give a fair representation also of the 1993 HERA data.

GRV(HO) [19] is a prediction. The PDF"s are evolved from a very low scale of $\mathrm{Q}_{0}{ }^{2}=$ $0.3 \mathrm{GeV}^{2}$, starting from 'valence like' quark and gluon distributions. In this case much of the steep rise in $F_{2}$ at low $x$ is generated dynamically through gluon bremsstrahlung from quarks and quark pair creation from gluons by the GLAP evolution in $Q^{2}$. Next- 
to-leading order is used. Up to $\mathrm{Q}^{2}=50 \mathrm{GeV}^{2}$ the GRV(HO) curves include the improved treatment of the charm contribution [20] w- hich is sizeable at low $Q^{2}$ and decreases $F_{2}$ by about $10 \%$. Good agreement with the HERA data is observed.

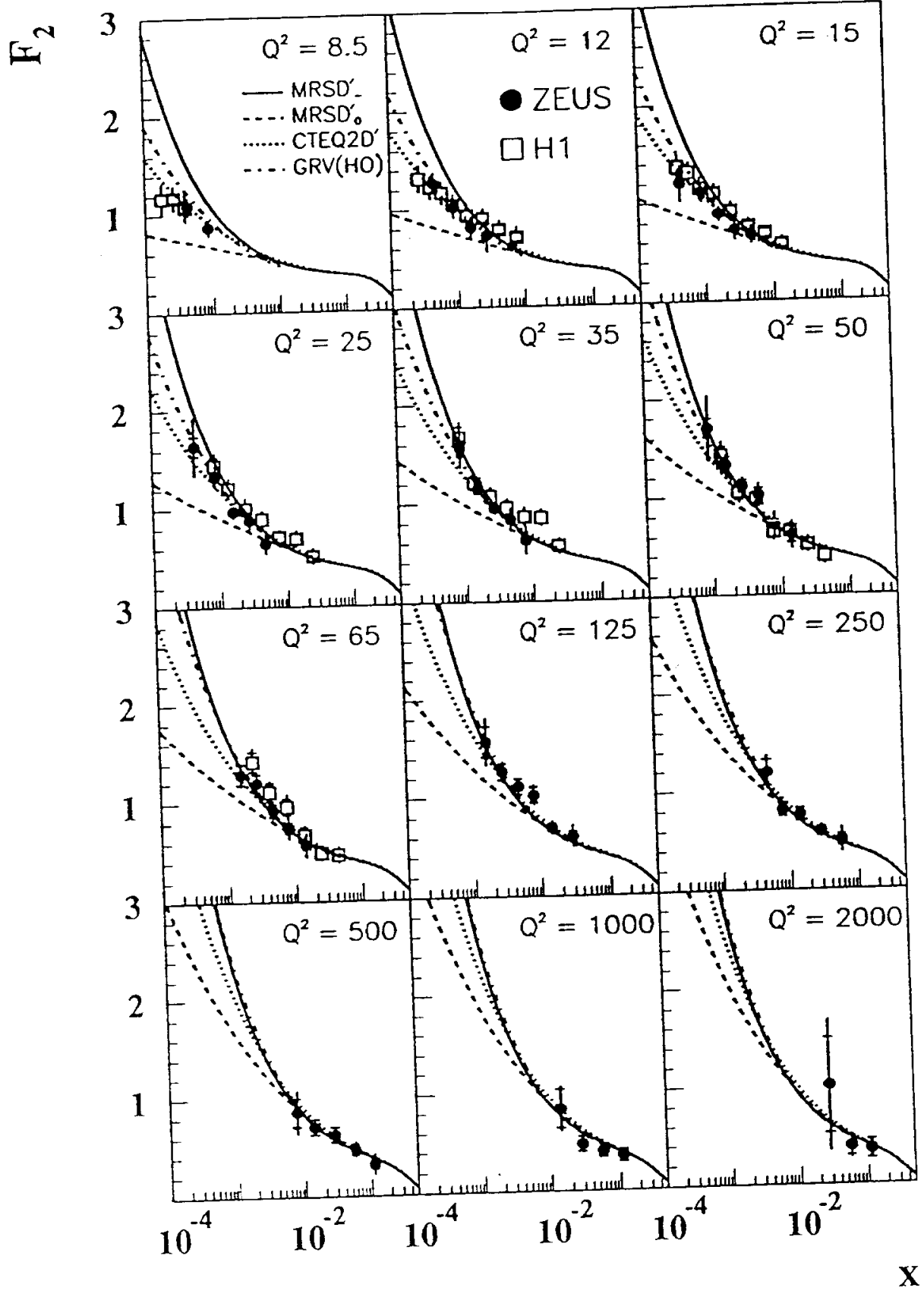

Figure 11. The proton structure function $\mathrm{F}_{2}$ as a function of $\mathrm{x}$ at fixed $\mathrm{Q}^{2}$ as determined by $\mathrm{H} 1$ and ZEUS. The H1 data are preliminary. The error bars show the statistical and systematic errors added in quadrature. The curves were calculated with the parton densities MRSD-', MRSDo' and CTEQ2D'; also shown is the GRV(HO) prediction. 


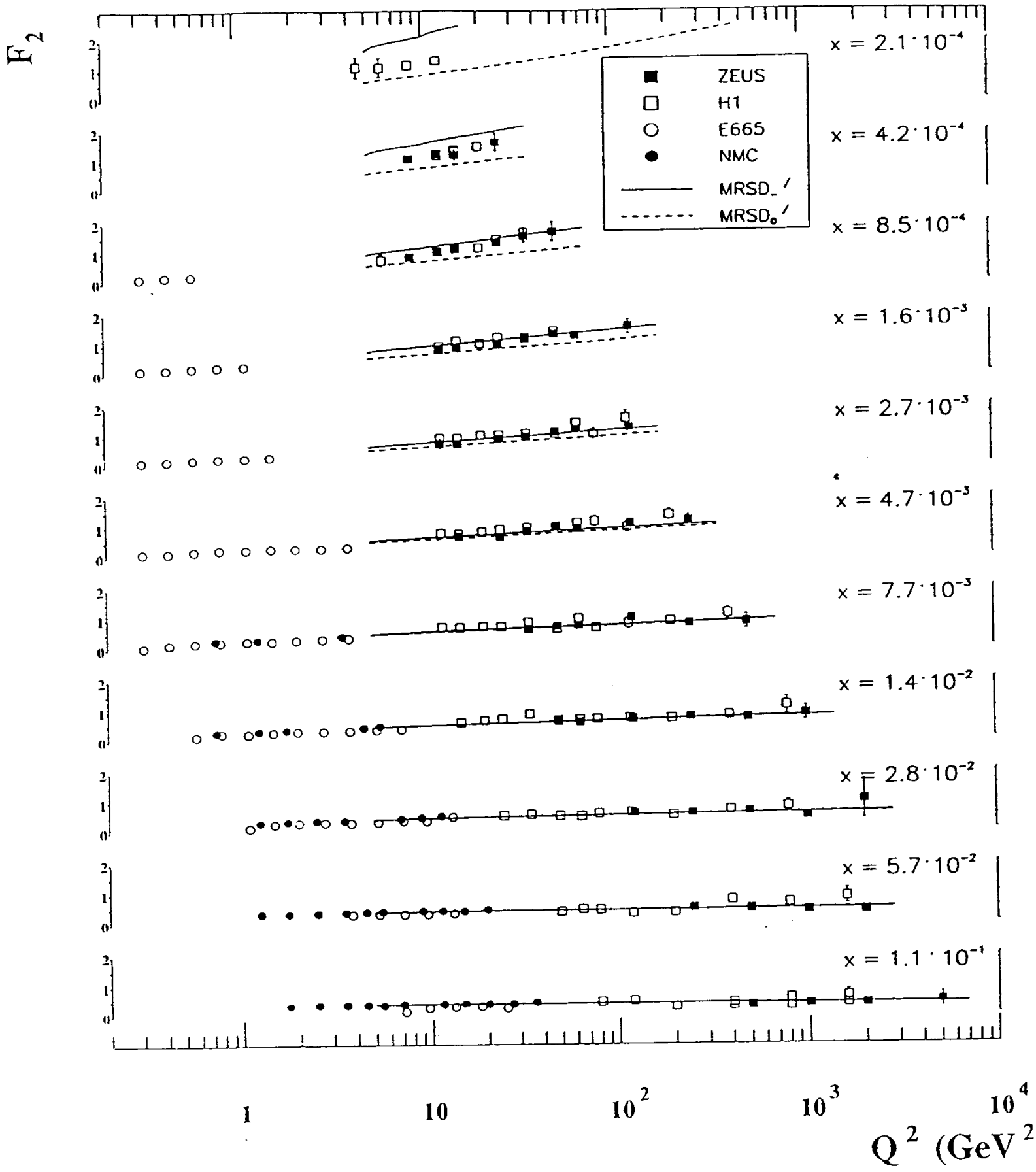

Figure 12. The proton structure function $F_{2}$ as a function of $Q^{2}$ at fixed $x$ as determined by $H 1$ and ZEUS. The H1 data are preliminary. The error bars show the statistical and systematic errors added in quadrature. Also shown are data from NMC and E665, the latter being preliminary. The curves were calculated with the parton densities MRSD.' and MRSDo'. 


\section{3 $\quad \mathbf{F}_{2}$ and $\sigma_{\text {tot }}\left(\gamma^{*} \mathbf{p}\right)$}

Neglecting contributions from $Z^{O}$ exchange the DIS cross section can be expressed in terms of the flux of virtual photons times the total cross section, $\sigma_{\text {tot }}\left(\gamma^{*} p\right)$, for scattering of the virtual photon on the proton [21]. $\sigma_{\text {to }}\left(\gamma^{*} p\right)$ is defined in terms of the cross sections for the scattering of transverse and longitudinal photons, $\sigma_{\mathrm{T}}$ and $\sigma_{\mathrm{L}}$, respectively, by

$\sigma_{\text {tot }}\left(\gamma^{*} p\right) \equiv \sigma_{T}\left(x, Q^{2}\right)+\sigma_{L}\left(x, Q^{2}\right)$.

The photon flux is well defined provided the lifetime of the virtual photon is large compared to the interaction time or $x \ll 1 /\left(2 m_{p} R_{p}\right)$ where $m_{p}$ is the mass of the proton and $R_{p}=$ $4 \mathrm{GeV}^{-1}$ its radius [22]. For $\mathrm{x}<10^{-2}$ this condition is well satisfied. The expression for $F_{2}$ in terms of $\sigma_{\mathrm{T}}$ and $\sigma_{\mathrm{L}}$ is

$$
F_{2}\left(x, Q^{2}\right)=\frac{Q^{2}(1-x)}{4 \pi^{2} \alpha}\left[\sigma_{T}\left(x, Q^{2}\right)+\sigma_{L}\left(x, Q^{2}\right)\right]
$$

At small $x$ the expression can be rewritten in terms of the virtual photon proton c.m. ener$\mathrm{gy} W$ to give

$\sigma_{\text {tot }}\left(\gamma^{*} p\right)=\frac{4 \pi^{2} \alpha}{Q^{2}} F_{2}\left(W, Q^{2}\right)$.

The connection between $F_{2}$ and $\sigma_{\text {tot }}\left(\gamma^{*} p\right)$ has been investigated by others for fixed target DIS data, for example [23].

Relation (4) was used by ZEUS [9] to determine $\sigma_{\text {tot }}\left(\gamma^{*} p\right)$ from the $F_{2}$ data. Figure 12 shows $\sigma_{\text {tot }}\left(\gamma^{*} p\right)$ as a function of $W$ from 50 to $280 \mathrm{GeV}$ for $\mathrm{Q}^{2}$ between 8.5 and 125 $\mathrm{GeV}^{2}$. At all these $\mathrm{Q}^{2}$ values $\sigma_{\text {tot }}\left(\gamma^{*} \mathrm{p}\right)$ increases with increasing $W$ as could have been inferred from the $x$ dependence of $F_{2}$. Given the size of the errors the increase is compatible with a linear rise (the straight lines in Fig. 13 are drawn to guide the eye) but other functions of $\mathrm{W}$ give equally good descriptions of the trend. The magnitude of the slope $\operatorname{d\sigma }_{\text {tot }}\left(\gamma^{*} p\right) / d W$, at fixed $Q^{2}$ is dominated by the factor $Q^{2}$ in equation (3). This is shown in Fig. 14 where $Q^{2} \cdot \sigma_{\text {tot }}\left(\gamma^{*} p\right)$ is plotted as a function of $\mathrm{W}$ for the data with $\mathrm{Q}^{2}$ between 8.5 and $125 \mathrm{GeV}^{2}$. The data cluster along a narrow band.

The rise with $\mathrm{W}$ is in marked contrast to that of the total cross section for real photon proton scattering (Fig. 15) which exhibits only a slow rise between the fixed target regime $(\mathrm{W}<20 \mathrm{GeV})[24]$ and the data from HERA near $\mathrm{W}=200 \mathrm{GeV}[25,26]$ compatible with $W^{0.16}$ [27]. A total cross section that rises rapidly with $W$ is also unlike the total $\overline{p p}$ cross section. This is the only hadron cross section measured in the hundred to thousand $\mathrm{GeV}$ range and its energy dependence can be described by a term proportional to $\mathrm{W}^{0.16}[27]$. By expressing $\mathrm{F}_{2}$ in terms of $\sigma_{\text {tot }}\left(\gamma^{*} p\right)$ one sees from another angle that with deep inelastic scattering at low $x$ the HERA experiments have entered a new regime of physics [29].

Using the optical theorem, $\operatorname{Im~} A\left(\gamma^{*} p\right)$ $\sigma_{\text {tot }}\left(\gamma^{*} p\right)$, where $A\left(\gamma^{*} p\right)$ is the invariant amplitude for $\gamma^{*} p$ elastic scattering, one sees that if $\sigma_{\text {tot }}\left(\gamma^{*} p\right)$ rises linearly with $W$ at a fixed value of $Q^{2}$ the elastic cross section will rise $\sim W^{2}$ and will violate eventually unitarity unless it is damped above some $\mathrm{W}=\mathrm{W}_{\text {crit }}$ by parton saturation [30]. Whether saturation can be detected in the HERA regime is an exciting question.

\section{CONCLUDING REMARKS}

The advance into the region of low $x$ at large $Q^{2}$ has shown a new regime of physics. The proton structure function $F_{2}$ rises rapidly as $x$ tends to zero signalling a rapid increase of the density of partons in the proton. The fact that the model of GRV $[15,19]$ is able to give 


\section{ZEUS 1993 DATA}
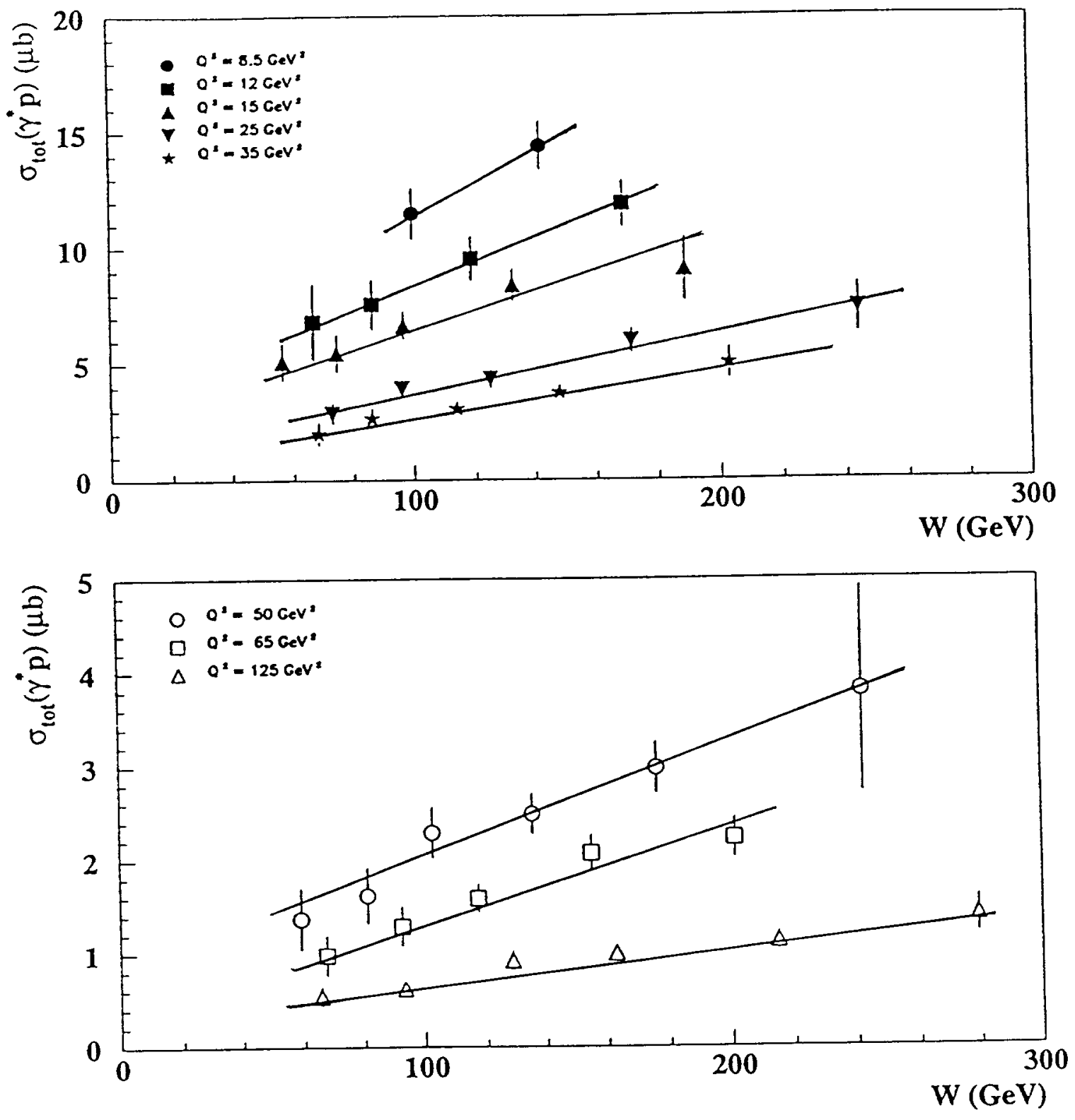

Figure 13. The virtual photon proton cross section $\sigma_{t o t}\left(\gamma^{*} p\right)$ as a function of $W$ for fixed $Q^{2}$ between 8.5 and $125 \mathrm{GeV}^{2}$ determined by ZEUS. The straight lines are drawn to guide the eye. 


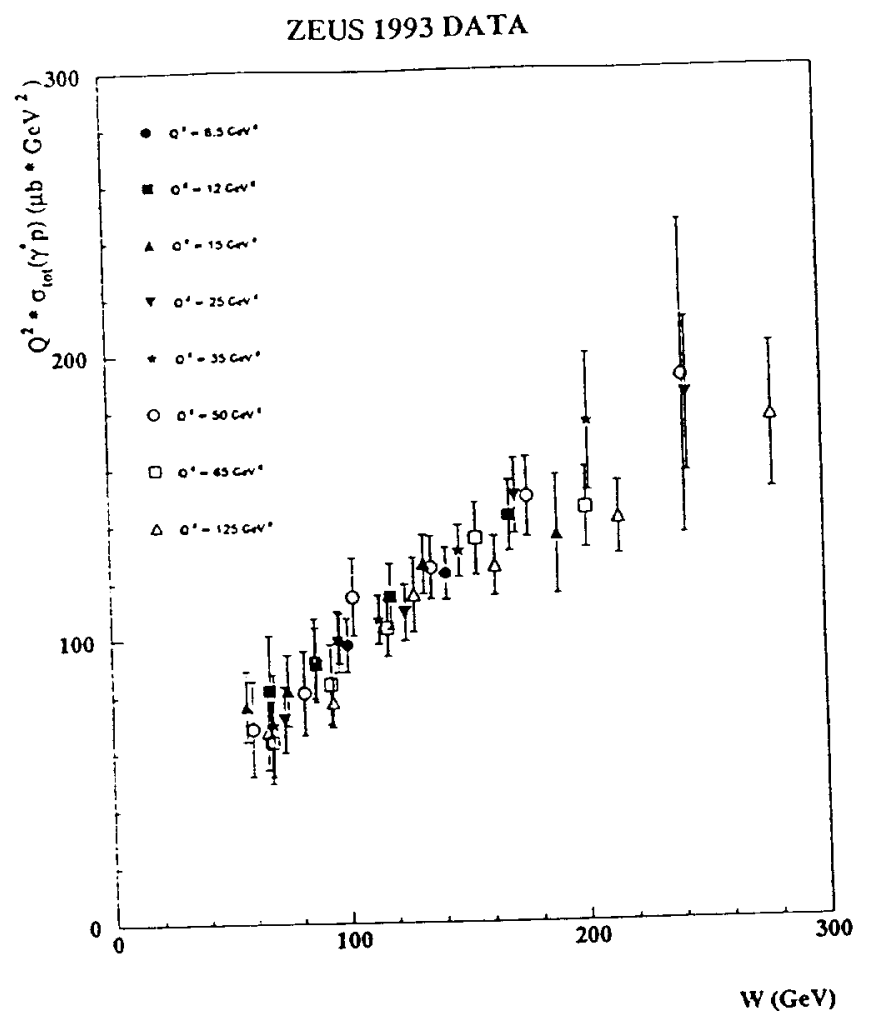

Figure 14. $\mathrm{Q}^{2} \cdot \sigma_{\mathrm{tot}}\left(\gamma^{*} \mathrm{p}\right)$ as a function of $\mathrm{W}$ for fixed $\mathrm{Q}^{2}$ between 8.5 and $125 \mathrm{GeV}^{2}$. (From ZEUS).

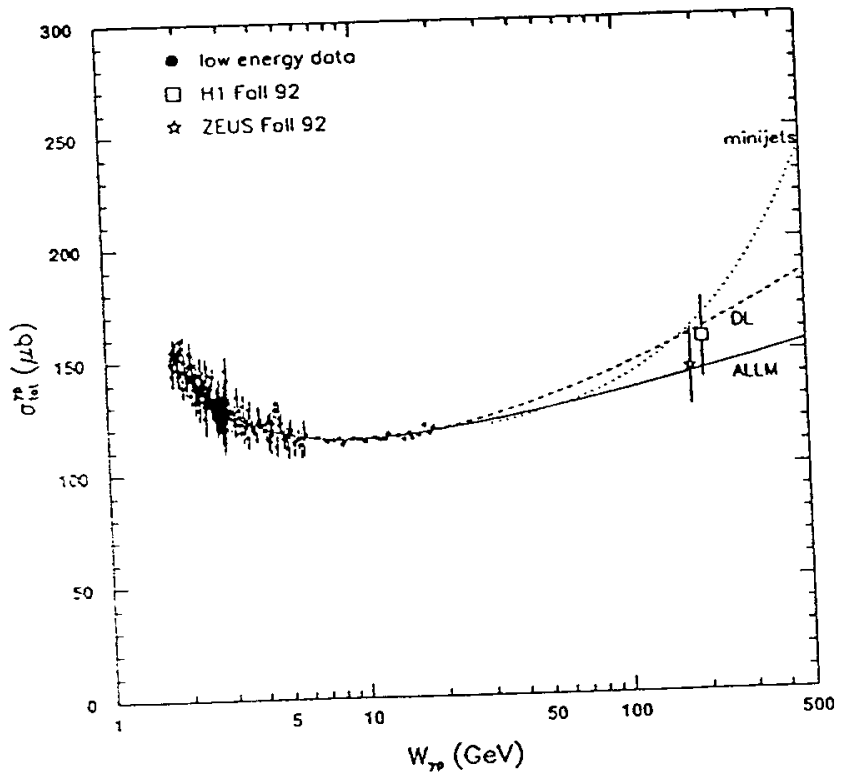

Figure 15. The total $r p$ cross section (at $Q^{2}=0$ ) as a function of $\mathrm{W}$ as measured below $18 \mathrm{GeV}$ [24] and near $200 \mathrm{GeV}$ by H1 [25] and ZEUS [26]. The solid curves show the predictions of DL [27] and ALLM [28]. 
a good account of the $F_{2}$ measurements suggests that perhaps all of the parton density increase is radiatively generated. The extension of the $F_{2}$ measurements down to $Q^{2}$ values of $\sim 1 \mathrm{GeV}^{2}$ in order to determine the turn-on of the rise at low $x$ as a function of $\mathrm{Q}^{2}$ will provide an important test of this idea.

The rapid rise of $F_{2}$ towards low $x$ is equivalent to a rapid increase of $\sigma_{\text {tot }}\left(\gamma^{*} p\right)$ with c.m. energy W. In conjunction with the optical theorem this implies that the elastic cross section $\sigma\left(\gamma^{*} p \rightarrow \gamma^{*} p\right)$ rises even faster with $W$ and should eventually violate unitarity. Parton saturation is expected to avert the clash with unitarity. Since $\sigma\left(\gamma^{*} p \rightarrow \gamma^{*} p\right)$ is expected to be very small at HERA energies the problem with unitarity in elastic scattering would become acute only at much higher energies. However, the large rapidity gap events observed recently in deep inelastic scattering by ZEUS [31], and confirmed by H1 [32], exhibit a diffractive-like behaviour similar to that of elastic scattering. Since the production rate for this type of events is substantial, a measurement of the cross section and its $W$ dependence might give a clue on parton saturation already at HERA energies.

The results presented here were obtained with an integrated luminosity corresponding to about one percent of the yearly design luminosity. Over the next two to three years, the luminosity should increase to its design value of $50 \mathrm{pb}^{-1}$ per year. This will benefit all of HERA physics and will be most important for charged current processes and for the in vestigation of the weak currents at large $Q^{2}$.

\section{ACKNOWLEDGEMENTS}

I am grateful to my colleagues from the two HERA experiments for providing me with their data and for many discussions. Dr. M. Lancaster has been particularly helpful with putting the data from the two experiments together. I want to thank Prof. J. Whitmore for a critical reading of the manuscript.

\section{REFERENCES}

1. H1 collaboration, I. Abt et al., Nucl. Phys. B407 (1993) 515.

2. ZEUS collaboration, M. Derrick et al., Phys. Lett. B316 (1993) 412.

3. F. Jacquet and A. Blondel, Proc. Study of an ep facility in Europe, ed. U. Amaldi, 79/48 (1979), p. 391.

4. S. Bentvelsen, S. Engelen and P. Kooijman, HERA Workshop 1991, ed. W. Buchmüller and G. Ingelman, Vol. 1, p. 23.

5. H1 collaboration, $T$. Ahmed et al., DESY 94-012 (1994).

6. ZEUS collaboration, preliminary data presented by D. Krakauer and G. Wolf, at the Intern. Workshop on DIS and Related Subjects, Feb. 1994, Eilat, Israel; S. Nickel, PhD Thesis (Univ. Hamburg) 1994.

7. A.D. Martin, W.J. Stirling and R.G. Roberts, Phys. Rev. D47 (1993) 867; Phys. Lett. B306 (1993) 145; RAL93-77/ DTP93/86(1993).

8. H1 collaboration, preliminary data presented by V. Brisson and J. Feltesse at the 27th Intern. Conf. High Energy Physics, July 1994, Glasgow, UK

9. ZEUS collaboration, M. Derrick et al., DESY 94 - 143 (1994).

10. V.N. Gribov and L.N. Lipatov, Sov. J. Nucl. Phys. 15 (1972) 438, 675; L.N. Lipatov, Sov. J. Nucl. Phys. 20 (1974) 181; G. Altarelli and G. Parisi, Nucl. Phys. 126 (1977) 297.

11. NMC collaboration, P. Amaudruz et al., Phys. Lett. B295 (1992) 159.

12. E665 collaboration, data presented by A.V. Kotwal at the VIth Renc. Blois, France, June 1994.

13. For a nonasymptotically free vector theory: V.N. Gribov and L.N. Lipatov, Yad. Fiz. 15 (1972) 781; Sov. J. Nucl. Phys. 15 (1972) 438. 
14. For an asymptotically free vector theory: A. De Rujula et al., Phys. Rev. D10 (1974) 1649.

15. M. Glück, E. Reya and A. Vogt, Z. Phys. C48 (1990) 471; Z. Phys. C53 (1992) 127.

16. L.N. Lipatov, Sov. J. Nucl. Phys. 23 (1976) 338.

17. CTEQ collaboration, J. Botts et al., Phys. Lett. B304 (1993) 159 and W.K. Tung, priv. communication.

18. A.D. Martin, R.G. Roberts and W.J. Stirling, Univ. Durham DTP/94/34, RAL-94-055 (1994).

19. M. Glück, E. Reya and A. Vogt, Phys. Lett. B306 (1993) 391.

20. M. Glück, E. Reya and M. Stratmann, Univ. Dortmund DO-TH 93/20 (1993).

21. L.N. Hand, Phys. Rev. 129 (1963) 1834; S.D. Drell and J.D. Walecka, Ann. Phys. (N.Y.) 28 (1964) 18; F.J. Gilman, Phys. Rev. 167 (1968) 1365.

22. B.L. Joffe, V.A. Khoze and L.N. Lipatov, 'Hard Processes', North Holland (1984) p185.

23. A. Levy and U. Maor, Phys. Lett. 182B (1986) 108.
24. S.I. Alekhin et al., CERN-HERA 87-01 (1987) and references given therein.

25. H1 collaboration, T. Ahrned et al., Phys. Lett. B297 (1992) 205.

26. ZEUS collaboration, M. Derrick et al., DESY 94-032 (1994), to be published in Z. Phys. C.

27. A. Donnachie and P.V. Landshoff, Nucl. Phys. B244 (1984) 322; P.V. Landshoff, Nulc. Phys. B (Proc. Suppl.) 18C (1990) 211; Z. Phys. C61 (1994) 139.

28. H. Abramowicz et al., Phys. Lett. 269 (1991) 465.

29. That total cross sections for off-shell particles might rise rapidly with $W$ has been considered in C. Lopez and F.J. Yndurain, Phys.. Rev Lett. 44 (1980) 1118.

30. see e.g. L.V. Gribov, E.M. Levin and M.G. Ryskin, Phys. Rep. 100 (1983) 1.

31. ZEUS collaboration, M. Derrick etal., Phys. Lett. B315 (1993) 481; ibid. B332 (1994) 228; DESY 94-117 (1994).

32. H1 collaboration, T. Ahmed et al., DESY 94-133 (1994). 